\title{
Avaliação da diversidade da entomofauna em função de técnicas de amostragem, em pomar, no sul do Brasil
}

\begin{abstract}
Eva Carla Lobo
Natália Rampelotto Santi

Instituto Federal Farroupilha, Campus Alegrete, Brasil

Luciane Ayres-Peres

Instituto Federal Farroupilha, Campus São Vicente do Sul, Brasil

Thiago Della Nina Idalgo

Universidade Federal de Santa Maria, Brasil e Instituto Federal Farroupilha, Campus

Jaguari, Brasil

DOI: https://doi.org/10.31492/2184-2043.RILP2018.35/pp.69-82

Resumo

O objectivo deste trabalho foi analisar a diversidade da entomofauna encontrada em pomar de árvores frutíferas em função de diferentes técnicas de amostragem, no Sul do Brasil. Foram utilizadas três técnicas para amostragem: caça-mosca, técnica da bandeja colorida e armadilhas do tipo pitfall, em frutíferas de: Citrus sinensis, Ficus carica e Rubus fruticosus. Foi registrada uma abundância total de 12.943 indivíduos, distribuídos em nove ordens e 46 famílias. As ordens com maior abundância foram Diptera (60,07\%), Coleoptera (31,07\%) e Hymenoptera (7,47\%). Considerando os índices de diversidade em função das armadilhas, houve maior diversidade nas bandejas $(\mathrm{H}=0,9454)$ do que na pitfall $(\mathrm{H}=0,7788)$ e armadilha caça-mosca $(\mathrm{H}=0,6306)$. Tal facto ocorreu, possivelmente, em função uma menor dominância das distintas ordens nas bandejas. Cada método considera um comportamento dos insectos, seja alimentar ou de locomoção, e se complementam para um levantamento que se aproxime do real em pomar de frutíferas.
\end{abstract}

Palavras-chaves: armadilhas; bandeja Colorida; caça-mosca; entomologia; pitfall.

\section{Abstract}

The aim of this study was to analyze the entomofauna diversity found in the orchard of fruit trees, in function of different techniques to capture, in Southern Brazil. Three traps were used for insect collection, hunting-fly, colored tray technique and pitfall. Were choose the follow fruit trees: Citrus sinensis, Ficus carica, and Rubus fruticosus. An abundance of 12,943 individuals was recorded, distributed in nine orders and 46 families. The most abundant orders were Diptera (60.07\%), Coleoptera (31.07\%) and Hymenoptera (7.47\%). Considering the diversity index in function of the traps, there were a bigger diversity on the trays $(\mathrm{H}=0.9454)$ than on pitfall $(\mathrm{H}=0.7788)$ and hunting-fly traps $(\mathrm{H}=0.6306)$. That occurs due, possibly, a smaller dominance in the trays. Each method considers an insect behavior, of feed or of locomotion, and complete each other to a realistic assessment in a fruit trees orchard.

Keywords: colored tray; entomology; fly-hunting; pitfall; traps. 


\section{Introdução}

A entomologia é a ciência que estuda os insectos sob todos os aspectos, estabelecendo suas relações com os seres humanos, plantas e outros animais (Gallo et al., 2002). Os insectos constituem o táxon mais numeroso e diverso do Reino Animal e são considerados o grupo mais diverdificado entre os Arthropoda, compreendendo o maior número de espécies deste filo, além de abranger aproximadamente 75\% das espécies de animais conhecidas (Barbosa, 2008). Esses animais vivem na Terra há cerca de 300 milhões de anos e, durante este tempo, evoluíram em diversas direcções para se adaptarem a quase todos os tipos de habitats.

O sucesso adaptativo dos insectos ocorre por diversos factores, dentre os quais, a evolução do voo concedeu-lhes uma vantagem sobre os demais invertebrados terrestres (Ruppert, Fox, \& Barnes, 2006), sobretudo na dispersão, fuga dos predadores, acesso ao alimento e condições ambientais ideais (Del-Claro \& Torenzan-Silingardi, 2012). Na sua evolução, adquiriram características que lhes beneficiaram quanto aos problemas de suprimento de alimento, protecção contra inimigos, adaptação a condições ambientais específicas e organização social (Barbosa, 2008). Sua importância está relacionada com seus hábitos e as funções que exercem na natureza tais como: reciclagem da matéria orgânica; dispersão de plantas; alimento para uma grande variedade de animais, controle de populações de animais e plantas (Barbosa, 2008).

Alguns insectos são considerados espécies-chave e a sua existência é essencial para a manutenção de seus ecossistemas (Gullan \& Cranston, 2012), como a polinização de plantas que é um fenómeno essencial para a manutenção da biodiversidade, entretanto, outros podem se tornar pragas agrícolas ou causar danos à saúde de humanos e animais agindo como parasitas ou vectores de doenças (Barbosa, 2008).

Essas pragas agrícolas compreendem populações de organismos capazes de causar danos às plantas, seus produtos e subprodutos. $\mathrm{O}$ dano pode afectar o rendimento do produto ou sua qualidade, através do consumo directo dos tecidos ou órgãos da planta, frutos ou sementes, sucção de seiva, transmissão de doenças, competição por espaço e por nutrientes (Santos, 2011).

Os insectos têm-se mostrado indicadores apropriados para avaliação de ecossistemas, devido à sua diversidade, ciclo e capacidade de adaptação, geralmente em curto espaço de tempo (Silveira-Neto \& Monteiro, 1995). Nesse contexto, as espécies frutíferas destacam-se como locais onde se concentra a maior riqueza desses animais (Silva, 2013).

Os estudos sobre riqueza de espécies em diferentes estruturas vegetais são ferramentas básicas para levantamentos iniciais da diversidade regional (Barboza 
\& Uchoa, 2010) Para realização de levantamentos são utilizadas armadilhas, que consistem em qualquer dispositivo contendo um atractivo para os animais e disposto de uma forma que evite a saída dos mesmos (Triplehorn \& Johnson, 2011). O uso de armadilhas é mais fácil e menos oneroso para levantamento de insectos (Melo, Moreira, \& Silva, 2011). Uma armadilha pode ser definida como o processo mecânico, físico ou químico que captura um organismo, constituída de dois dispositivos básicos: o de atracção e o de captura (Nakano \& Leite, 2000).

As armadilhas caça-mosca, como as do modelo McPhail, podem ser úteis para o monitoramento de alterações de diferentes componentes dessa diversidade, como os parâmetros populacionais, seja perante condições ambientais distintas, em resposta a impactos de processos naturais ou de actividades humanas (Nascimento et al., 2003). Armadilhas do tipo caça-moscas têm grande importância na avaliação da diversidade para alguns grupos de insectos que são atraídos pelo odor do actrativo (Steyskal, 1977). Na técnica da bandeja colorida os insectos são atraídos pela cor, através de uma bandeja ou pratos rasos de plástico ou metal colorido, com água, colocados ao nível do solo (Williams, Minckley, \& Silveira, 2011).

A técnica do pitfall, ou armadilhas de queda, são específicas para animais que caminham sobre o solo (Almeida, Ribeiro-Costa, \& Marinoni, 1998). Esta consiste em um método passivo de coleta, que depende da atividade do organismo, fornecendo uma estimativa aproximada do número total de espécies de uma comunidade, além de ser uma metodologia simples e barata para estudos ecológicos (Silva \& Carvalho, 2000).

Com base no exposto, o objectivo deste trabalho foi analisar a diversidade da entomofauna encontrada em pomar de árvores frutíferas em função de diferentes técnicas de amostragem, no Sul do Brasil.

\section{Material e métodos}

O levantamento da entomofauna foi realizado no Instituto Federal de Educação, Ciência e Tecnologia Farroupilha (IFFar), Campus Alegrete - RS/Brasil, no período de 29 de Setembro a 25 de Novembro de 2015. O pomar de frutíferas do Campus foi escolhido para realização do experimento, entre as espécies cultivadas, três delas foram eleitas: a laranjeira Citrus sinensis cv. valência, a figueira Ficus carica cv. pingo-de-mel, e a amoreira Rubus fruticosus cv. tupy.

O pomar possui uma área de 4,8 hectares. As cultivares de laranjeira foram plantadas no espaçamento de 4,0 x 6,0 m totalizando sua área em 1,9 ha, as figueiras possuem espaçamento de 2,5 x 6,0 m e possuem uma área de 0,078 ha e as amoreiras tem somente uma linha com espaçamento de 1,5 x 6,0 m. 


\subsection{Técnicas de amostragem}

Foram utilizados três tipos de armadilhas para o levantamento, armadilhas tipo caça-moscas, bandeja colorida de fundo amarelo e armadilha de queda tipo pitfall. As armadilhas do tipo caça-moscas foram confeccionadas com garrafas PET (Polietileno Tereftalato). Cada qual com quatro furos de $02 \mathrm{~cm}$ de diâmetro, localizados na parte mediana da garrafa PET. Os furos foram distanciados $6 \mathrm{~cm}$ entre si, cada espécie de planta no pomar envolvida no experimento recebia uma armadilha. A solução atractiva utilizada foi suco de uva natural (Aliança Premier $\left.\mathrm{BAG}^{\circledR}\right)$, não diluído, depositada no fundo da armadilha, com o auxílio de um funil a partir da boca da garrafa. As armadilhas foram instaladas no centro de cada linha do pomar das frutíferas de laranja, amora e figo, a uma altura equivalente ao terço mediano da copa (Figura 1).

Para as armadilhas de fundo colorido foram utilizadas bandejas plásticas de 40 x $25 \mathrm{~cm}$, que tiveram seu fundo e laterais internos forrados com papel contact de cor amarela. As bandejas foram dispostas na fileira de cada frutífera, entre duas plantas abaixo do dossel sobre o solo. As mesmas receberam uma solução de etileno glicol 20\%, para que os insectos atraídos ficassem retidos, em quantidade suficiente para cobrir o fundo e ter uma profundidade de aproximadamente três centímetros. A cada colecta dos insectos capturados a solução foi substituída.

As armadilhas do tipo pitfall foram confeccionadas com garrafas PET de dois litros, com 10,5 cm de diâmetro, cortadas ao meio de modo que as aberturas estivessem a $20 \mathrm{~cm}$ de altura. As mesmas foram dispostas no centro da fileira de plantas com o bocal ao nível do solo, contendo álcool $70 \%$ até cerca de cinco centrímetros. Para evitar o que a precipitação diluísse o álcool, cada garrafa teve a parte superior, o gargalo, mantidos sobre elas, apoiados por tiras de plástico da própria garrafa. Após toda a colecta dos insectos capturados o álcool foi reposto da mesma maneira descrita anteriormente.

\subsection{Coleta e triagem do material biológico}

Durante o fase experimental, foram realizadas ao total, quatro colectas. Foram padronizadas três armadilhas de cada tipo para cada frutífera, as colectas para este período foram feitas a cada sete dias (1 vez por semana). Os insectos capturados nas armadilhas foram colectados com o auxílio de dois crivos plásticos e um pincel, acondicionados em potes de plástico de $30 \mathrm{ml}$ contendo álcool a $70 \%$, rotulados com a data da colecta, nome da armadilha e planta do pomar, em seguida levados ao Laboratório de Biologia do IFFar - Campus Alegrete para triagem.

Posteriormente, os insectos foram triados, quantificados e identificados até o nível taxonómico de família, com o uso de chaves para o grupo (Gallo et al., 
2002; Triplehorn \& Johnson, 2013), sendo então etiquetados e acondicionados em recipientes de $5 \mathrm{ml}$ contendo solução de álcool 70\%.

\subsection{Análise dos dados}

Os animais foram quantificados, os dados foram submetidos a ANOVA (Análise de Variância) e sendo assim comparada a abundância da entomofauna entre as três espécies frutíferas do pomar (Teste de Kruskal-Wallis, KW).

Para verificar a diversidade, considerando as três espécies de frutíferas (em todas as técnicas de coleta) e para avaliar a eficiência de cada técnica amostral (caça-mosca $x$ bandeja colorida x pitfall) foram obtidos os índices de Shannon-Wiener $(H)$, Dominância de Simpson $(D)$ e Equitabilidade $(J)$.

O índice de Shannon-Wiener $(H)$, considera igual peso entre as espécies raras e abundantes (Magurran, 1989). Esse índice estima a diversidade de variáveis categóricas em uma população, avaliando os aspectos da riqueza e equitabilidade, os quais dizem respeito ao número de categorias da variável em questão e às proporções de cada uma destas, respectivamente (Ayres et al., 2007). No Índice de Simpson $(D)$ atribui-se maior riqueza para a população com maior número de categorias em relação às outras (Silva, 2010). A Equitabilidade $(J)$ se dá à população com maior homogeneidade nas proporções de suas categorias (Silva, 2010), indica se diferentes espécies possuem abundâncias (número de indivíduos) semelhantes ou divergentes (Gallo et al., 2002). O índice de dominância expressa a relação entre o número de indivíduos de uma determinada espécie e o número de indivíduos de todas as espécies encontradas (Raga, 2005).

As análises estatísticas foram realizadas com programa BioEstat (Ayres et al., 2007) e Past 3.1 (Hammer et al., 2001), com significância de 5\%.

\section{Resultados e discussão}

$\mathrm{Na}$ área do estudo foi registrada uma abundância de 12.943 indivíduos, distribuídos em 46 famílias e nove ordens de Insecta (Tabela 1). Dentre todos os taxa identificados, as ordens que apresentaram maior abundância foram Diptera (60,07\%), Coleoptera (31,07\%) e Hymenoptera $(7,47 \%)$.

Nas três frutíferas verificaram-se as três famílias que apresentaram maior abundância. No pomar de laranja (C. sinensis cv. Valência), foram encontrados $68,85 \%$ dos indivíduos de Drosophilidae (Diptera), 15,25\% de Staphylinidae (Coleoptera) e 5,26\% de Erotilydae (Coleoptera). Na amoreira (R. fruticosus cv. tupy) 50,82\% dos indivíduos foram de Drosophilidae, 21,00\% de Staphylinidae e 9,41\% de Erotylidae. Quanto ao pomar de figueira (F. carica cv. Pingo-de-mel), 
$34,20 \%$ dos indivíduos foram Drosophilidae, 34,62\% Staphylinidae e 12,26\% de Erotylidae (Tabela 1).

Tabela 1. Número de indivíduos capturados por armadilha: caça-mosca, bandeja amarela e pitfall, nas frutíferas: laranjeira, amoreira e figueira, em pomar do IFFar - Campus Alegrete/RS Brasil, no período de Setembro a Novembro de 2015.

\begin{tabular}{|c|c|c|c|c|c|c|c|c|c|c|}
\hline \multirow[b]{2}{*}{ Orden } & \multirow[b]{2}{*}{ Famútia } & \multicolumn{3}{|c|}{ Laranjeirz } & \multicolumn{3}{|c|}{ Ancréra } & \multicolumn{3}{|c|}{ Frguera } \\
\hline & & caga-mosca & Bandga anarela & Figull & Caga-mosca & Bandga anarela & Fquall & Cagamosca & Bandga amarela & Aquall \\
\hline \multirow{2}{*}{ Blattodea } & Blartidae & & & 01 & & 01 & & & & \\
\hline & Blustelidse & 20 & . & . & 23 & . & . & 19 & . & . \\
\hline \multirow{11}{*}{ Coleoptera } & Staphylinidute & 910 & . & 58 & 630 & 01 & 07 & 1229 & 2 & . \\
\hline & Erentidae & . & & 07 & . & 02 & . & 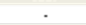 & . & \\
\hline & Eruchidae & . & 01 & a1 & . & 02 & 12 & . & . & 5 \\
\hline & Coccinellidats & 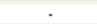 & . & 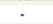 & 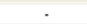 & . & : & . & 1 & . \\
\hline & Erotylidae & 282 & . & 52 & 285 & 01 & . & 432 & . & 4 \\
\hline & Cermbycidse & 06 & . & . & 02 & . & . & 4 & . & . \\
\hline & Chrysomelidae & . & 02 & 01 & . & . & . & . & . & . \\
\hline & Elaterides & 1 & . & . & as & . & . & . & . & . \\
\hline & Passalidae & . & . & 04 & . & . & . & . & . & . \\
\hline & Meloidxe & - & . & - & - & - & - & 1 & - & - \\
\hline & Curculionidre & - & - & 04 & as & - & - & - & - & - \\
\hline \multirow{14}{*}{ Diptera } & Chironomidae & 06 & . & . & 17 & . & . & 9 & . & . \\
\hline & Drosophilidue & 4371 & . & . & 1544 & . & . & 1216 & . & . \\
\hline & Mycetophilidae & 23 & . & . & $s_{1}$ & . & . & 20 & . & . \\
\hline & Otridase & 32 & . & . & 47 & . & . & 29 & . & . \\
\hline & Tachinidse & 79 & & - & 101 & - & - & 111 & - & . \\
\hline & Sarcophagidae & 25 & . & . & 16 & . & . & 10 & . & . \\
\hline & Culliphoridse & 10 & . & . & 06 & . & . & 8 & . & . \\
\hline & Conopidae & . & . & - & & - & - & 1 & . & . \\
\hline & Muscidare & . & . & . & 03 & . & - & . & . & . \\
\hline & Culicidse & & . & . & 01 & . & . & . & . & . \\
\hline & Sciaridae & 02 & . & - & 02 & . & . & . & . & . \\
\hline & Lonchaeidare & & . & . & 02 & & . & . & . & . \\
\hline & Syphhidare & 02 & . & . & 02 & 1 & . & . & . & . \\
\hline & Tephritidase & 29 & . & . & . & . & . & . & . & . \\
\hline \multirow{5}{*}{ Hemiptera } & Pertatomidre & . & . & - & . & . & . & 1 & . & . \\
\hline & Membracidae & . & . & . & . & 02 & . & : & . & 1 \\
\hline & Aleyrodidut & . & . & . & . & . & . & . & . & 1 \\
\hline & Cicadellidat & . & 02 & . & . & 01 & . & . & . & . \\
\hline & Miridae & . & . & - & . & . & . & 1 & . & . \\
\hline \multirow{9}{*}{ Hymenoptera } & Scelionidate & 02 & . & . & . & 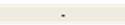 & . & i & . & \\
\hline & Scoliidat & . & . & . & . & 01 & . & : & 2 & . \\
\hline & Vespidae & 06 & & 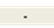 & 13 & & & 22 & & \\
\hline & Mostillidae & & 02 & . & & 03 & 01 & & 1 & $?$ \\
\hline & Encytidae & 99 & 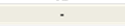 & . & 32 & 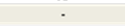 & 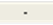 & 179 & . & . \\
\hline & Ichneumonidse & 275 & . & . & 73 & . & . & 149 & . & . \\
\hline & Figtidae & 01 & . & . & . & $\cdot$ & . & 2 & . & . \\
\hline & Fommicidae & . & . & - & 41 & 04 & 06 & 2 & 15 & 26 \\
\hline & $\begin{array}{l}\text { Apidare } \\
\text { Nothidare }\end{array}$ & & . & 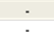 & & 01 & ${ }^{\circ}$ & 1 & 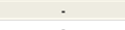 & \\
\hline 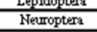 & $\begin{array}{l}\text { Noctuidaee } \\
\text { Crysopidae }\end{array}$ & $\begin{array}{l}32 \\
01\end{array}$ & : & : & $\begin{array}{l}89 \\
022\end{array}$ & : & : & $\begin{array}{c}40 \\
1\end{array}$ & $\therefore$ & : \\
\hline Demmaptera & Spongiophoridat & . & . & . & . & . & . & . & 1 & . \\
\hline \multirow[t]{2}{*}{ Orthoptera } & $\begin{array}{c}\text { Proscopididae } \\
\text { Tentrigidise }\end{array}$ & : & : & : & : & . & . & . & . & 1 \\
\hline & $\begin{array}{l}\text { TetaL } \\
\text { Todae }\end{array}$ & 6214 & 07 & 128 & 2992 & 20 & 26 & 3488 & 22 & 46 \\
\hline
\end{tabular}

\subsection{Análise comparativa da diversidade e abundância em função da téc- nica de amostragem}

Considerando os índices de diversidade em função das armadilhas (somados os dados das três frutíferas), houve maior diversidade, através do Índice de Shannon-Wiener $(H)$, nas bandejas amarelas $(H=0,9454)$ do que na pitfall $(H=0,7788)$ e na armadilha caça-mosca $(H=0,6306)$. Tal fato ocorreu, possivelmente, em função da menor dominância $(D=0,1878)$ nas bandejas e melhor distribuição $(J=0,8038)$ em relação às outras técnicas amostrais $(D=0,2215 ; J=0,6795$, no pitfall e $D=0,3724 ; J=0,4189$, no caça-mosca) (Tabela 2). 
Tabela 2. Valores obtidos dos Índices de Shannon-Wiener $(H)$, Simpson $(D)$ e Equitabilidade $(J)$, através dos Programas Bioestat e Past. Os mesmos foram obtidos por técnica de captura caça-mosca, bandeja amarela e pitfall, pelo somatório das frutíferas, laranjeira, amoreira e figueira, em pomar do IFFar - Campus Alegrete/RS - Brasil, no período de Setembro a Novembro de 2015.

\begin{tabular}{cccc}
\hline & Caça-mosca & Bandeja amarela & Pitfall \\
\hline Índice de Shannon-Wiener - H & 0.6306 & 0.9454 & 0.7788 \\
Dominância de Simpson-D & 0.3724 & 0.1878 & 0.2215 \\
$\quad$ Equitabilidade- J & 0.4189 & 0.8038 & 0.6795 \\
\hline
\end{tabular}

Quando se utilizou a armadilha do tipo caça-moscas verificou-se que não houve diferença significativa de riqueza entre as frutíferas, encontrou-se 22 famílias na laranjeira, 24 em amoreira e 24 em figueira. Quando considerada a diversidade, através do Índice de Shannon-Wiener, observou-se essa foi menor na laranjeira $(H=0,4828)$ do que na amoreira $(H=0,7084)$ e figueira $(H=0,7167)$, sendo que os índices máximos de diversidade hipotética para essas espécies foram de $H=1,324, H=1,3802$ e $H=1.3802$, respectivamente. A equitabilidade foi menor na laranjeira $(J=0,3597)$ seguida da figueira $(J=0,5193)$ e da amoreira $(J=0,5133)$ (Tabela 3). Em relação à abundância, capturou-se um total de 6.214 indivíduos na laranjeira, 2.992 na amoreira e 3.488 na figueira $(K W=0,3479, \mathrm{p}=0,8403)$.

Pelo método de colecta com bandeja colorida, verificou-se riqueza de quatro famílias na laranjeira, 12 na amoreira e seis em figueira. Porém, quando considerada a diversidade, através do índice de Shannon-Wiener, observou-se uma diversidade maior na amoreira $(H=1,0187)$ do que na laranjeira $(H=0,5871)$ e figueira $(H=0,4858)$, sendo que os índices máximos de diversidade hipotética para essas espécies foram de $H=1,0792, H=0,6021$ e $H=0.7782$, respectivamente. Comparando-se a equitabilidade essa foi menor na figueira $(\mathrm{J}=0,6243)$ do que na laranjeira $(\mathrm{J}=0,9751)$ e na amoreira $(\mathrm{J}=0,9440)$ (Tabela 3$)$. A abundância encontrada através da técnica da bandeja colorida foi de sete indivíduos na laranjeira, 20 na amoreira e 22 na figueira $(\mathrm{KW}=5,8322, \mathrm{p}=0,0541)$.

Tabela 3. Valores obtidos dos Índices de Shannon-Wiener $(H)$, Simpson $(D)$ e Equitabilidade $(J)$, através dos Programas Bioestat e Past. Os mesmos foram obtidos por técnica de captura caça-mosca, bandeja amarela e pitfall, em cada uma das frutíferas, laranjeira, amoreira e figueira, em pomar do IFFar - Campus Alegrete/RS - Brasil, no período de Setembro a Novembro de 2015.

\begin{tabular}{ccccccccccc}
\hline & \multicolumn{3}{c}{ Caca-mosca } & \multicolumn{3}{c}{ Bandejaamarela } & \multicolumn{3}{c}{ Pitfall } \\
\cline { 2 - 10 } & Laranjeira Amoreira Figueira Laranjeira Amoreira Figueira Laranjeira Amoreira Figueira \\
\hline Indice de Shannon-Wiener-H & 0.4828 & 0.7084 & 0.7167 & 0.5871 & 1.0187 & 0.4858 & 0.5272 & 0.5098 & 0.6061 \\
Dominância de Simpson-D & 0.5208 & 0.3233 & 0.2668 & 0.2653 & 0.1100 & 0.4876 & 0.3755 & 0.3402 & 0.3639 \\
Equitabilidade-J & 0.3597 & 0.5133 & 0.5193 & 0.9751 & 0.9440 & 0.6243 & 0.5838 & 0.8467 & 0.6711 \\
\hline
\end{tabular}


Através do pitfall, foram obtidas oito famílias na laranjeira, quatro em amoreira e oito em figueira. O índice de Shannon-Wiener, foi de $H=0,5272$ para laranjeira, $H=0,5098$ para amoreira e $H=0,6061$ na figueira. Os índices máximos de diversidade hipotética para essas espécies foram de $H=0,9031$ para laranjeira e figueira e $H=0,6021$ para a amoreira. A equitabilidade foi menor na laranjeira $(J=0,5838)$ seguida da figueira $(J=0,6711)$ e da amoreira $(J=0,8467)$ (Tabela 3$)$. Foram coletados, no total, 128 indivíduos na laranjeira, 46 na figueira e 26 na figueira $(\mathrm{KW}=1,7374, \mathrm{p}=0,4195)$.

Segundo Ricklefs (1996) o Índice $H$ é uma medida logarítmica da diversidade ponderada pela abundância relativa de cada uma, enquanto a riqueza uma simples contagem do número de famílias. Analisando o índice de diversidade, que leva em conta a abundância relativa e a equitabilidade de uma comunidade, percebe-se que no pomar de laranjeira, ocorreu um maior número de indivíduos em relação às demais espécies (Tabela 1), porém sua diversidade foi menor, provavelmente em função da distribuição heterogênea da abundância com poucas famílias detendo um maior número de indivíduos.

A armadilha caça-mosca se torna atractiva para os insectos que reconhecem o odor do atrativo alimentar utilizado. Os insetos ao entrarem no interior da armadilha, voam em direção à solução e acabam morrendo afogados (Gallo et al., 2002), porém, esse é um método de amostragem seletivo, atraindo a grupos restritos de invertebrados (Pinheiro-Machado \& Silveira, 2006).

A armadilha de solo pitfall é um dos métodos de coleta mais empregados e mais eficientes na captura de invertebrados de solo (Aristophanous, 2010; Gullan \& Cranston, 1994; Ward \& Larivière, 2004), esse tipo de armadilha pode capturar diversos tipos grupos de animais, como macroinvertebrados que habitam o solo até mamíferos.

\subsection{Análise comparativa da diversidade e abundância em função das ár- vores frutíferas}

Analisando a diversidade em função das três espécies de frutíferas, verificou-se que a figueira foi a que apresentou maior número de famílias, 33, em relação à amoreira, 32 e à laranjeira, 29. Foi verificado o Índice $H$ de 0,7509 para a figueira, $H=0,7338$ para a amoreira e $H=0,505$ para a laranjeira (Tabela 4 ). De acordo com Carauta (2002), a figueira possui uma vasta diversidade de plantas espontâneas que podem servir de atractivo para invertebrados.

Porém, quando se considera a abundância a laranjeira apresentou maior abundância com 6.349 indivíduos, seguida da figueira e da amoreira com 3.556 e 3.038 indivíduos, respectivamente $(\mathrm{KW}=0,1923, \mathrm{p}=0,9083)$. A equitabilidade 
foi menor na laranjeira $(J=0,3453)$ seguida da amoreira $(J=0,4875)$ e da figueira $(J=0,4945)$ e a dominância apresentou tendência inversa, sendo o menor índice o da figueira $(D=0,2575)$ seguido da amoreira $(D=0,3249)$ e o maior o da laranjeira $(D=0,5024)$ (Tabela 4).

Tabela 4. Valores obtidos dos Índices de Shannon-Wiener $(H)$, Simpson $(D)$ e Equitabilidade $(J)$, através dos Programas Bioestat e Past. Os mesmos foram obtidos por frutífera laranjeira, amoreira e figueira; pelo somatório das técnicas caça-mosca, bandeja amarela e pitfall, em pomar do IFFar - Campus Alegrete/RS - Brasil, no período de Setembro a Novembro de 2015.

\begin{tabular}{cccc}
\hline & Laranjeira & Amoreira & Figueira \\
\hline Índice de Shannon-Wiener - H & 0.5050 & 0.7338 & 0.7509 \\
Dominância de Simpson-D & 0.5024 & 0.3149 & 0.2575 \\
Equitabilidade- J & 0.3453 & 0.4875 & 0.4945 \\
\hline
\end{tabular}

Em Diptera, observou-se uma maior frequência de Drosophilidae, possivelmente, devido ao fato de ter ocorrido fermentação do suco de uva, pois, as moscas dessa família constituem pragas domésticas quando há frutas presentes em decomposição e fungos. A maior incidência de Drosophilidae ocorreu no pomar de laranjeira, provavelmente pela presença de frutas em decomposição sob a copa das árvores, pois na amoreira e figueira ainda não havia frutos no período do experimento. Por esse mesmo motivo, de somente laranjeira possuir frutos, explica-se o fato de que somente nas armadilhas caça-mosca colocadas nessa espécie frutífera tenham sido encontrados indivíduos da família Tephritidae, a qual pertencem as moscas-das-frutas. Estas são reconhecidamente pragas de citros e podem estar presentes em pomares atacando as frutas.

A quantidade de Drosophilidae nas amostras é similar às descritas em outros estudos, como o de Pasini (2013), que utilizando a solução de suco de uva, obteve $36,6 \%$ de indivíduos dessa família coletados em pomar de figo no Sul do Brasil (Agudo - RS, Brasil). O mesmo demonstrado por Bortoli (2014) que obteve $43,1 \%$ dos indivíduos capturados dessa família em pomares de diversas espécies na região serrana do Rio Grande do Sul (Sul do Brasil).

A grande incidência de besouros de Staphylinidae nas amostras pode ser explicada também pela fermentação do suco na armadilha caça-mosca, visto que estes besouros vivem em uma grande variedade de ambientes, mas são vistos com maior frequência em materiais em decomposição. Também foi esta a família mais abundante capturada pela armadilha pitfall em laranjeira, possivelmente pelo mesmo motivo supra citado da presença de frutos das laranjeiras em decomposição no solo. A quantidade de Erotylidae pode estar relacionada também com a decomposição e crescimento de fungos na solução na armadilha com atrativo 
suco de uva, e no solo na pitfall. Insetos desta família se encontram em locais onde há maior abundância de fungos decompositores.

No estudo de Manfio et al. (2007), utilizando armadilhas do tipo pitfall, em um fragmento de Floresta Estacional Decidual (vegetação pertencente ao Bioma Mata Atlântica), também houve uma maior abundância de Staphylinidae. Esta família costuma viver sob pedras e outros objetos do solo, ao longo de córregos, fungos, montes de folhas e ninhos de árvores. Os hábitos alimentares de Staphylinidae são bastante variados, a maioria das espécies é predadora de outros insetos e invertebrados, mas muitas delas são saprófagas e se alimentam de matéria orgânica em decomposição, o que justifica o grande número de exemplares amostrados, pois ambas as armadilhas utilizadas são de solo, e as frutíferas apresentavam frutas em decomposição no período amostral. No estudo de Petroni (2008), utilizando material em decomposição como atrativo para captura de insetos, foi encontrada uma grande quantidade de besouros da família Staphylinidae (7,8\%), enquanto de Erotylidae não houve quantidade expressiva. Já em outro trabalho, Silva (2010) encontrou uma quantidade de $26,3 \%$ de insetos Erotylidae em armadilhas tipo McPhail no Pantanal. Ganho \& Marinoni (2005) verificaram a quantidade de 41 indivíduos desta família, em estudo realizado em um ambiente com interferência antrópica (de setembro de 1999 a agosto de 2000), utilizando armadilhas para a captura de invertebrados.

A grande incidência de coleópteros pode ser explicada devido ao aumento de temperatura registrada durante a primavera (no Sul do Brasil), sendo que as mesmas apresentam variações entre $25^{\circ} \mathrm{C}$ a $30^{\circ} \mathrm{C}$. Corroborando os resultados obtidos no presente estudo, no qual, as amostragens também foram realizadas na primavera. Os números expressivos dos indivíduos da ordem Hymenoptera na armadilha tipo caça-moscas representados pelas famílias Vespidae, Encyrtidae, Braconidae e Figitidae (parasitoides e predadores), provavelmente decorrem da existência de diversos insetos na solução que podem ser presas ou hospedeiros de muitas espécies destas famílias, que são conhecidas por atuarem no controle de insetos-praga. Outro fator pode ser a fonte de alimentação que o atrativo alimentar suco de uva oferece a esses indivíduos, que se alimentam também de pólen e néctar na fase adulta (Gallo et al., 2002; Gullan \& Cranston, 2012).

Gattelli (2006) verificou uma grande quantidade de indivíduos himenópteros da família Braconidae em pomar de Citrus, em uma porcentagem de 66,7\%, relatando serem esses parasitoides frequentemente associados à mosca-das-frutas. Corroborando com os estudos de Oliveira (2008) nos quais também houve uma grande frequência dessa família em pomares de pinha. Segundo Silva \& Brito 
(2015), Encyrtidae é um agente de controle biológico de insetos-praga, ocorrendo em pomares de frutíferas, como em citros.

Nas armadilhas bandeja amarela e pitfall nas frutíferas amoreira e figueira a ordem Hymenoptera, apresentou maior abundância da família Formicidae, que segundo Triplehorn \& Johnson, esta ordem ocorre praticamente em todas as partes e habitats terrestres. No trabalho de Morais, Barcellos \& Redaelli (2006), realizado durante a primavera, a ordem Hymenoptera foi a que registrou maior número de indivíduos, todos pertencentes à Formicidae. Malerbo-Souza \& Souza (2005), em levantamentos de visitantes florais, utilizando a técnica pitfall, a ordem com mais indivíduos foi Hymenoptera e a família com maior número de exemplares foi Formicidae. Segundo Costa (2012), o predomínio dos formicídeos pode ser explicado pela dominância exercida por esses insetos na maioria dos ecossistemas terrestres, estando distribuídos em praticamente todas as regiões do planeta.

Figura 1. Ilustração do posicionamento da armadilha tipo caça-moscas no terço mediano da copa das frutíferas do pomar do IFFar - Campus Alegrete/RS - Brasil.

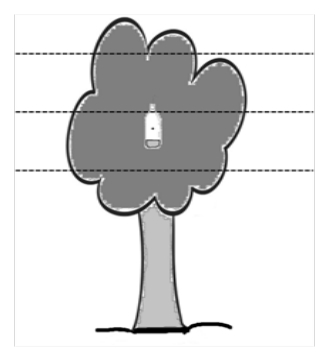

\section{Conclusão}

Com o presente estudo, observou-se a diversidade da entomofauna em uma área de pomar no Sul do Brasil. Destaca-se que cada método considera um comportamento dos insetos, seja alimentar ou de locomoção, e se complementam para um levantamento que se aproxime do real em pomar de frutíferas. Em estudos de levantamentos/inventários faunísticos, destaca-se a importância do uso de diferentes métodos de colecta que possibilitem a amostragem de animais com os mais variados hábitos, dessa maneira, chegando a estimativas mais realistas sobre a diversidade local.

\section{Agradecimentos}

Os autores são gratos aos funcionários e equipe diretiva do Instituto Federal de Educação, Ciência e Tecnologia Farroupilha - Campus Alegrete por todas as facilidades e colaborações na execução desse trabalho. 


\section{Referências}

Almeida, L., Ribeiro-Costa, C., \& Marinoni, L. (1998). Manual de coleta, conservação, montagem e identificação de insetos. São Paulo: Editora Holos.

Aristophanous, M. (2010). Does your preservative preserve? A comparison of the efficacy of some pitfall trap solutions in preserving the internal reproductive organs of dung beetles. ZooKeys, v.34, n.1, p. 1-16;

Ayres, M; Ayres Júnior, M; Ayres, D. L. \& Santos, A. A. (2007). BioEstat: Aplicações estatísticas nas áreas das ciências Biomédicas, Belém-Pará.

Barbosa, O. (2008). Entomofauna de solo em áreas de vegetação nativa e de cultivo de cana-de-açúcar no município de União Piauí (Dissertação em Agronomia). Universidade Federal do Piauí, Teresina.

Barboza, L., \& Uchoa, M. (2010). Diversidade de insetos capturados em armadilha Mcphail no Pantanal Sul mato grossense. (pp. 22-30.) Interbio v.4, n1.

Bortoli, L. C. (2014). Interações tritróficas entre moscas-das-frutas (Diptera: Tephritidae), seus hospedeiros e parasitoides (Hymenoptera) e avaliação de atrativos para o monitoramento na Região da Serra Gaúcha - RS, EMBRAPA, Pelotas;

Carauta, J., \& Diaz, B. E. (2002). Figueiras no Brasil. Rio de Janeiro: Editora UFRJ.

Costa, E. (2012). Entomofauna associada a cultura da melancia no semiárido do Rio Grande do Norte. (Dissertação de pós-graduação em Fitotecnia pela U. F. Rural do Semi-árido). Mossoró-RN;

Del-Claro, K., \& Torenzan-Silingardi, H. (2012). Ecologia das interações plantas-animais: uma abordagem ecológica evolutiva. Rio de Janeiro: Techinal Books.

Gallo, D.; Nakano, O.; Neto, S. S.; Baptista, G. C.; Filho, E. B.; Parra, J. R.;..;Omoto, C. (2002). Entomologia agrícola, Piracicaba: Editora FEALQ;

Ganho, N. G., \& Marinoni, R. C. (2005). A diversidade inventarial de Coleoptera (Insecta) em uma paisagem antropizada do Bioma Araucária. Departamentos de Zoologia. Universidade Federal do Paraná- PR;

Gattelli, T. (2006). Moscas frugivoras (Diptera: Tephritoidea) e parasitoides associados a mirtáceas e laranjeira 'céu' em Montenegro e Harmonia RS, UFRGS, Porto Alegre-RS;

Gullan, P., \& Cranston, P. (2012). Os insetos: um resumo de Entomologia. São Paulo: Editora Roco;

Hammer, O., Harper, D.A.T., \& Ryan, P.D. (2001). PAST: Paleontological Statistic software package for education and data analysis. Paleontologia Eletronica v.4, n.1, p. 1-9. https://palaeo-electronica.org/2001_1/past/issue1_01.htm;

Magurran, A. E. (1989). Diversidade Ecologico y su Medicion. Espanish: Edíciones vedro;

Malerbo- Souza, D. T., \& Souza. F.F. (2005). Entomofauna visitante e produção de frutos em melancia (Citrullus lanatus Thunb.) - Curcurbitaceae. Acto Scientiarum Agronomy, v. 27, n. 3, p. 454;

Manfio, D., Dal Berto, A. C., \& Di Mare, R. A. (2007). Inventário da ocorrência de

Coleoptera em duas localidades no municipio de Santa Maria, RS. Anais do VIII

Congresso de Ecologia do Brasil, Caxambu; 
Melo, L., Moreira, A., \& Silva, F. (2001). Armadilha para monitoramento de insetos. Embrapa Meio Ambiente: Jaguariuna: São Paulo;

Morais, R. M. De, Barcellos, A. Redaelli, L. R. ( 2006). Insetos predadores em copas de Citrus deliciosa (Ritaceae) sob manejo orgânico no Sul do Brasil. Porto Alegre: Iherirgia;

Nakano, O., \& Leite, C. (2000). Armadilhas para insetos. Biblioteca de Ciências Agrárias Luiz de Queiros, v.7. Piracicaba: FEALQ;

Nascimento, R. P.; Morini, M. S. C.; Silva, R. R. (2003). Diversidade de formigas de serapilheira (Hymenoptera: Formicidae) em áreas de Mata Atlântica e reflorestada com Pinus elliotti (Pinaceae), em uma região do alto do Tietê (Salesópolis, SP). Anais do XVI Simpósio de Mirmecologia, Florianópolis, Brasil, p.370-373;

Oliveira, P. R. (2008). Levantamento da entomofauna na cultura da Pinha (Anonna squamosa L.) em Janaúba, Norte de Minas Gerais, Unimontes. Janaúba-MG.

Pasini, M. (2013). Soluções atrativas para o monitoramento populacional de Zaprionus indianus (Diptera: Drosophilidae) em pomar de figo. e-ISSN 1983-4063 - www.agro.ufg.br/pat Pesq. Agropec. Trop., Goiânia;

Petroni, M. D. (2008). Diversidade da familia de coleoptera em diferentes fragmentos florestais no municipio de Londrina, PR - Brasil. 2008. 61 p. Dissertação (Mestrado em Ciências Biológicas)-Universidade Estadual de Londrina, Paraná;

Pinheiro-Machado, C., \& Silveira, F. A. (2006). Surveying and monitoring of pollinators in natural landscapes and in cultivated fields. In.: Fonseca, V. L. I., Saraiva, A. M., \& Jong, D. D. (Eds.). Bees as pollinators in Brazil: Assessing the status and suggesting best practices. Ribeirão Preto, Holos;

Raga, A. (2005). Incidência, monitoramento e controle de moscas-das-frutas na citricultura paulista. Laranja, Cordeirópolis, v.26, n.2, p.307-322;

Ricklefs, R.E. (1996). A Economia da Natureza. 3.ed. Rio de Janeiro: Guanabara Koogan;

Ruppert, E., Fox, R., \& Barnes, R. (2006). Invertebrate zoology. Belmont: Brooks; ColeThompson;

Santos, B. (2011). A origem e a importância dos insetos como praga das plantas cultivadas. Universidade Federal do Paraná - SCB;

Silva, A., \& Brito, J. (2015). Controle biológico de insetos-pragas e suas perspectivas para o futuro, Agropecuária Técnica, Volume 36 (1): 248-258, Versão Online ISSN 0100-7467, p. 248-258;

Silva, F. (2013). Plantas alimentares cultivadas nas roças Baniwa: mudanças e participação dos jovens. 2013. 122 f., il. Dissertação de Mestrado na Universidade de Brasília, Brasília;

Silva, L. B. (2010). Diversidade de insetos capturados em armadilha mcphail no pantanal sul-mato-grossense. Interbio v.4 n.1- ISSN 1981-3775, 22-30p. Mato Grosso do Sul;

Silveira-Neto, S., \& Monteiro, R. C. (1995). Uso da análise faunística de insetos na avaliação do impacto ambiental. Departamento de Entomologia. Piracicaba-SP: ESALQ/USP;

Steyskal, G. C. (1977). History and use of the McPhaill trap. The Florida Entomologist, Vol.60, N.1;

Silva, R., \& Carvalho, G. (2000). Ocorrência de insetos na cultura do milho em sistema de plantio direto, coletados com armadilhas de solo. Ciência Rural, v.30, n.2; 
Triplehorn, C. A., \& Johnson, N. F. (2011). Estudo dos insetos. Cengage Learning, São Paulo. $653 \mathrm{p}$.

Ward, D.F., \& Larivière, M.C. (2004). Terrestrial invertebrate surveys and rapid biodiversity assessment in New Zealand: lessons from Australia. New Zealand Journal of Ecology, V.28, n.1, p. 151-159;

Williams, N., Minckley, R., \& Silveira, F. (2001). Variation in native bee fauna and its implications for detecting community changes. Conservation Ecology. V.5, art.7.

Data receção: 01/06/2017

Data aprovação: $31 / 07 / 2017$ 